This assumption is the subject of intense scientific controversy. It derives in part from radiation biology studies and is based on the observation that many carcinogens are genotoxic that is, they cause direct damage to genes that may lead to cancer. Therefore, any level of exposure to a carcinogen might present some calculable risk of cancer. The risk is still related to the dose, as it is with noncarcinogenic effects.

Since typical human exposure levels to potentially carcinogenic pesticides are often several thousand times lower than the doses that have produced cancer in animal studies, calculation of carcinogenic risks requires extrapolation of the results of highdose animal studies to potential human effects at low levels of exposure. Scientists have adopted mathematical models to predict human effects based on animal effects.

Such models yield a value known as the cancer potency factor. Because a variety of different mathematical models exist, cancer potency factors vary widely depending upon the choice of the model and its assumptions. The most common model used is the linearized multistage model (see p. 26). This model assumes that a cell which may be a target for a carcinogenic chemical goes through a specific number of different stages and that the probability of a "hit" on the cell leading to cancer is stage-specific. At very low levels of exposure, the relationship between exposure level and excess cancers is linear; any finite level of exposure leads to some numerical value of excess cancers (above the background level in the population).
The NAS report recommended use of the two-stage clonal expansion model, itself a recent interpretation of the multistage model. This is a biologically based model of carcinogenesis that takes into account the special physiological characteristics of infants and children, such as the rapid rate of cell division and tissue growth. Biochemical events in infants and children, such as enzymes induction at different stages of development, may affect their response to pesticide residues. In addition, the NAS report recommended development of "pharmacokinetic" models for infants and children - that is, models that describe the child's unique physiological patterns of residue uptake and distribution, allowing for factors such as lactation and development. The report also recommended use of the so-called

\title{
Risk assessment defined
}

\section{Risk assessment}

Risk assessment is the characterization of the potential adverse health effects of human exposures to substances such as pesticide residues. It also includes characterization of uncertainties inherent in the process.

Risk assessments consist of the following steps:

Hazard identification, which is the determination of whether a particular chemical is or is not causally linked to particular health effects.

Dose-response assessment, which is the determination of the relation between the magnitude of exposure and the probability of occurrence of the health effects in question.

Exposure assessment, which is the determination of the extent of human exposure before or after application of regulatory controls.
Risk characterization, which is the description of the nature and often the magnitude of human risk, including attendant uncertainty.

In each step, a number of decision points occur where risk to human health can only be inferred from the available evidence and judgments must be made regarding the analytic approach to be taken.

\section{Related terms}

Conservative guideline: The term conservative has been used to describe inference options in hazard identification that increase the likelihood that a substance will be judged to be a significant hazard to human health.

Dose-response relationship: This is the relationship between the dose of a chemical substance received and the resulting physiological response. The larger the dose, the greater the effect. (Also, see Threshold.)

Risk management: Risk management is the process of evaluating alternative regulatory options and selecting among them. A risk assessment may be one of the bases of risk management.

Threshold: The minimum dose of a substance that produces a physiological effect. At extremely low doses, most chemical substances are detoxified by the body. Carcinogens are thought to be the exceptions. Because most cancer-causing substances are genotoxic - causing direct damage to genes - any one damaged cell could eventually give rise to a tumor, and any dose is hypothetically capable of causing cancer.

(Some definitions were adapted from Risk Assessment in the Federal Government: Managing the Process [National Academy Press, 1983].) -Editor 